\title{
Antibiotic Resistance of Helicobacter pylori Strains Isolated From Pediatric Patients in Southwest China
}

\author{
Juan $\mathrm{Li}^{1,2,3 \dagger}$, Jianjun Deng ${ }^{1,2+}$, Zhiling Wang ${ }^{1}$, Hong $\mathrm{Li}^{4 *}$ and Chaomin Wan ${ }^{1 *}$ \\ ${ }^{1}$ Department of Pediatrics, West China Second University Hospital, Sichuan University, Chengdu, China, ${ }^{2}$ Department of \\ Infection Control, West China Second University Hospital, Sichuan University, Chengdu, China, ${ }^{3}$ Key Laboratory of Birth \\ Defects and Related Diseases of Women and Children (Sichuan University), Ministry of Education, Chengdu, China, \\ ${ }^{4}$ West China Marshall Research Center for Infectious Diseases, Center of Infectious Diseases, West China Hospital, \\ Sichuan University, Chengdu, China
}

\section{OPEN ACCESS}

Edited by:

Rossella Grande,

University of Studies G. d'Annunzio

Chieti and Pescara, Italy

Reviewed by:

Mehmet Demirci,

Beykent University, Turkey

Mariana Carmen Chifiriuc,

University of Bucharest, Romania

*Correspondence:

Hong Li

42351160@qq.com

Chaomin Wan

wcm0220@sina.com

tThese authors have contributed equally to this work

Specialty section:

This article was submitted to Antimicrobials, Resistance and

Chemotherapy,

a section of the journal

Frontiers in Microbiology

Received: 27 October 2020 Accepted: 31 December 2020

Published: 26 January 2021

Citation:

Li J, Deng J, Wang Z, Li H and Wan C (2021) Antibiotic Resistance of Helicobacter pylori Strains Isolated

From Pediatric Patients in Southwest China.

Front. Microbiol. 11:621791. doi: 10.3389/fmicb.2020.621791
The number of antibiotics that are appropriate for Helicobacter pylori eradication in children is limited. Profiling regional or population-specific antibiotic resistance is essential in guiding the $H$. pylori eradication treatment in children. The aim of this study was to evaluate the antibiotic resistance in $\mathrm{H}$. pylori strains isolated from children and adolescents in Southwest China. Gastric biopsies from 157 pediatric patients with or without previous H. pylori eradication treatment were collected for $\mathrm{H}$. pylori culture. Susceptibility to amoxicillin (AML), clarithromycin (CLR), metronidazole (MTZ), levofloxacin (LEV), tetracycline (TET), furazolidone (FZD), and rifampicin (RIF) was determined by E-test or a disk diffusion assay. A total of 87 patients from three ethnic groups (Han/Tibetan/Yi) were $H$. pylori culture positive (55.4\%). The overall resistance rates were 55.2\% for CLR, 71.3\% for MTZ, 60.9\% for RIF, and $18.4 \%$ for LEV. No isolate was found to be resistant to AML, TET, and FZD. Among the 53 treatment-naiive pediatric patients, primary resistance rates to clarithromycin, metronidazole, levofloxacin, and rifampicin were 45.3, 73.6, 15.1, and 60.4\%, respectively. Among the 34 treatment-experienced patients, secondary resistance rates to clarithromycin, metronidazole, levofloxacin, and rifampicin were $70.6,67.6,23.5$, and $61.8 \%$, respectively. Isolates exhibiting simultaneous resistance to clarithromycin and metronidazole were 28.3 and $52.9 \%$ among the treatment-naïve and treatment-experienced patients, respectively. In conclusion, among pediatric patients in Southwest China, resistance rates were high for clarithromycin, metronidazole, levofloxacin, and rifampicin, whereas nil resistance was found to amoxicillin, tetracycline, and furazolidone. Our data suggest that the standard clarithromycin-based triple therapy should be abandoned as empiric therapy, whereas the bismuth quadruple therapy (bismuth/PPI/amoxicillin/tetracycline) would be suitable as first-line empiric treatment regimen for this pediatric population. Tetracycline and furazolidone may be considered for treating refractory $H$. pylori infections in adolescent patients.

Keywords: Helicobacter pylori, antimicrobial susceptibility testing, pediatric population, empiric therapy, rifampicin 


\section{INTRODUCTION}

Helicobacter pylori infection is a common bacterial infectious disease, the major cause of chronic active gastritis, peptic ulcers, and one of the most important risk factors for gastric cancer and mucosa-associated lymphoid tissue (MALT) lymphoma (Malfertheiner et al., 2017; Liu et al., 2018). It has been recommended that all adult patients with positive test of active $H$. pylori infection should be offered with $H$. pylori eradication therapy (Sugano et al., 2015; Chey et al., 2017; Malfertheiner et al., 2017). However, in comparison with adults, children, and adolescents with $H$. pylori infection rarely develop serious diseases or related symptoms (Jones et al., 2017; Liu et al., 2018). In addition, as $H$. pylori is usually acquired in childhood, the reinfection rate in children after successful H. pylori eradication is higher than in adults (Jones et al., 2017; Liu et al., 2018). Thus, the "test and treat" strategy is not suitable for $H$. pylori infection in children under 14 years of age (Liu et al., 2018). However, for children with peptic ulcer diseases, "test and treat" for $H$. pylori infection is required (Jones et al., 2017; Liu et al., 2018).

The number of antibiotics that are appropriate for $H$. pylori eradication in children is limited. The current recommended first-line $H$. pylori eradication regimens in children are mainly triple therapies consisting of a proton pump inhibitor (PPI) plus two antibiotics chosen from amoxicillin (AML), clarithromycin (CLR), and metronidazole (MTZ) for a duration of 14 days (Xu et al., 2015; Jones et al., 2017). The tetracycline (TET), furazolidone (FZD), and levofloxacin (LEV) that are appropriate for use in adults with $H$. pylori infection are relatively contradicted in Children due to potential side effects. LEV is usually contraindicated in children younger than 18 years due to potential severe side effects including tendon rupture. However, the use of TET can be considered in children older than 8 years (Xu et al., 2015; Jones et al., 2017). With the availability of FZD in China, the use of FZD may be considered in $>14$-year-old children with refractory $H$. pylori infections. ${ }^{1}$

Given the limited number of antibiotics that are appropriate for $H$. pylori eradication in children, and the worldwide increase of antibiotic resistance, the recent ESPGHAN/NASPGHAN guidelines have recommended antimicrobial susceptibility testing to guide the $H$. pylori eradication treatment in children (Jones et al., 2017). However, due to the fastidious culture of H. pylori and the lack of an easy and cost-effective testing method, antimicrobial susceptibility testing for $H$. pylori is almost universally unavailable in medical centers (Montes and PérezTrallero, 2017; Li et al., 2019; Tang et al., 2020a,b). Under such circumstances, profiling regional or population-specific antibiotic resistance patterns is of great importance in guiding the development of effective empiric treatment regimens. As the $H$. pylori antibiotic resistance profiles among children and adolescents in Southwest China is lacking, the aim of this study was to evaluate $H$. pylori strains isolated from this population for antibiotic resistance to CLR, MTZ, LEV, AML, TET, FZD, and rifampicin (RIF).

${ }^{1}$ https://www.dayi.org.cn/

\section{MATERIALS AND METHODS}

\section{Patients}

Patients with severe upper gastrointestinal symptoms referred to West China Second University Hospital from January 2019 to December 2019 for gastrointestinal endoscopy were considered for this study. Patients older than 18 years, with severe systemic illness, use of PPI or antibiotics in the last month; and previous gastric surgery were excluded. Informed consent was obtained from the pediatric patients' parents or guardians. This study was approved by the ethics committee of West China Second University Hospital, Sichuan University (HXEY2019011). A total of 157 pediatric patients from three different ethnic groups (Han, Tibetan, and Yi) were enrolled in this study.

\section{Helicobacter pylori Culture and Antimicrobial Susceptibility Testing}

Gastric biopsy samples collected from the gastric antrum and/ or corpus of the 157 pediatric patients were placed in sterile vials containing Brain Heart Infusion (BHI) medium supplemented with $20 \%$ glycerol and $5 \%$ new-born calf serum (NCS) and transferred on ice to the $H$. pylori laboratory at West China-Marshall Research Center for Infectious Diseases. The samples were stored at $-80^{\circ} \mathrm{C}$ until for culture of $H$. pylori. For H. pylori culture, frozen gastric samples were thawed, homogenized, and inoculated onto both non-selective commercial Columbia Blood Agar (CBA) plates (Auto bio, China) and selective CBA plates supplemented with Dent antibiotics (Oxoid, United Kingdom). Inoculated plates were incubated at $37^{\circ} \mathrm{C}$ under microaerobic conditions $\left(5 \% \mathrm{O}_{2}, 10 \% \mathrm{CO}_{2}\right.$, and $85 \%$ $\mathrm{N}_{2}$ ) generated using the Anoxomat Mark-II system (Mart Microbiology B.V., the Netherlands). Colonies displaying typical H. pylori morphology were confirmed by Gram-staining, positive urease-, catalase-, and oxidase-tests.

The isolated $H$. pylori strains were tested for susceptibility to CLR, MTZ, LEV, AML, TET, and RIF using the Epsilometer test strips (Liofilchem s.r.l, Italy), whereas susceptibility to FZD was determined using agar dilution method as previously described (Su et al., 2013).

According to the European Committee on Antimicrobial Susceptibility Testing (EUCAST), H. pylori resistance to CLR, MTZ, LEV, TET, and RIF were defined as the minimal inhibitory concentration $(\mathrm{MIC})>0.5 \mathrm{mg} / \mathrm{L},>8 \mathrm{mg} / \mathrm{L},>1 \mathrm{mg} / \mathrm{L},>1 \mathrm{mg} / \mathrm{L}$, and $>1 \mathrm{mg} / \mathrm{L}$, respectively (Kahlmeter et al., 2006). Base on a very recent clinical study (Graham et al., 2020), resistance to AML was defined as MIC $>0.25 \mathrm{mg} / \mathrm{L}$. Resistance to FZD was defined as MIC >2 mg/L (Kahlmeter et al., 2006; Su et al., 2013). The H. pylori strain NCTC 11637 was used for quality control.

\section{Statistics}

Data were statistically analyzed using SPSS version 24.0 (IBM Corporation, Armonk, NY, United States). Chi-square test $\left(\chi^{2}\right)$ or Fisher's exact test was used to investigate factors associated with antibiotic resistance among the pediatric patients. Differences were considered statistically significant with a value of $p<0.05$. 


\section{RESULTS}

Between January 2019 and December 2019, 157 children and adolescents who underwent gastrointestinal endoscopy were included in this study to have their gastric biopsies collected for $H$. pylori culture. A total of 87 patients were found to be $H$. pylori positive (55.4\%), 34 were girls and 53 were boys with a mean age of $10.9 \pm 2.8$ years (age range, 5-17 years). Fifty-three strains (60.9\%, 53/87) were isolated from treatment-naïve patients and the remaining $34(39.1 \%, 34 / 87)$ strains were isolated from treatmentexperienced patients. The main endoscopic findings among the $87 \mathrm{H}$. pylori culture-positive patients were gastritis/ duodenitis in $68(78.2 \%)$, followed by duodenal ulcer in 13 (14.9\%), gastric ulcer in three (3.4\%), and other diagnosis in three (3.4\%; Table 1 ).

TABLE 1 | Patient demographics and clinical characteristics.

\begin{tabular}{lc}
\hline Variables & Patients $(\boldsymbol{n}=\mathbf{8 7})$ \\
\hline Gender & \\
Male/female & $53 / 34$ \\
Race/ethnics & \\
Han & 59 \\
Tibetan & 22 \\
Yi & 6 \\
Mean age (years) & $10.9 \pm 2.8$ \\
Endoscopy findings & \\
Gastritis/duodenitis & 68 \\
Duodenal ulcer & 13 \\
Gastric ulcer & 3 \\
Other & 3
\end{tabular}

Among the 87 pediatric $H$. pylori strains with resistance profiled using the Epsilometer test strips (Figure 1), the overall antibiotic resistance rates were $55.2 \%$ (48/87) for CLR, $71.3 \%(62 / 87)$ for MTZ, 60.9\% (53/87) for RIF, and 18.4\% (16/87) for LEV. No isolate was found to be resistant to AML, TET, and FZD (Figure 2). Of the 53 strains isolated from patients without previous treatment, resistance rates to CLR, MTZ, LEV, and RIF were $45.3 \%(24 / 53)$, $73.6 \%(39 / 53), 15.1 \%(8 / 53)$, and $60.4 \%$ (32/53), respectively. Of these 53 strains, $3(5.7 \%)$ were susceptible to all the tested seven antibiotics, 12 were mono-resistant (22.6\%), 27 (50.9\%) were dual resistant, and the remaining 11 strains were resistant to three or more antibiotics, thus giving an MDR rate of $20.7 \%$ (Table 2).

Among the 34 strains isolated from patients with prior H. pylori treatment, resistance rates to CLR, MTZ, LEV, and RIF were $70.6 \%(24 / 34), 67.6 \%(23 / 34), 23.5 \%(8 / 34)$, and $61.8 \%(21 / 34)$, respectively (Figure 2 ). Of these 34 strains, only one strain $(2.9 \%)$ was susceptible to all the seven antibiotics, seven strains (20.6\%) were mono-resistant, 10 strains $(29.4 \%)$ were dual resistant, and the remaining 16 strains were MDR (47.0\%; Figure 2; Table 2). Of note, the total number of strains exhibiting simultaneous resistance to CLR and MTZ (including the dual and MDR strains) were 15 (28.3\%) from the 53 treatment-naïve patients, and 18 (52.9\%) from the $34 \mathrm{H}$. pylori treatment-experienced patients (Table 2).

Clarithromycin resistance rate in 5-11-year-old-group was comparable to that of the 12-17-year-old group (57.4 and 52.5\%, respectively; Table 3 ). No significant difference was found in resistance rate to $\mathrm{MTZ}, \mathrm{LEV}$, and RIF between the two age groups, either. Among the six strains isolated from Yi ethnic group (all were treatment naïve), one strain (16.7\%) was resistant to CLR, whereas five of them $(83.3 \%)$ were resistant to both
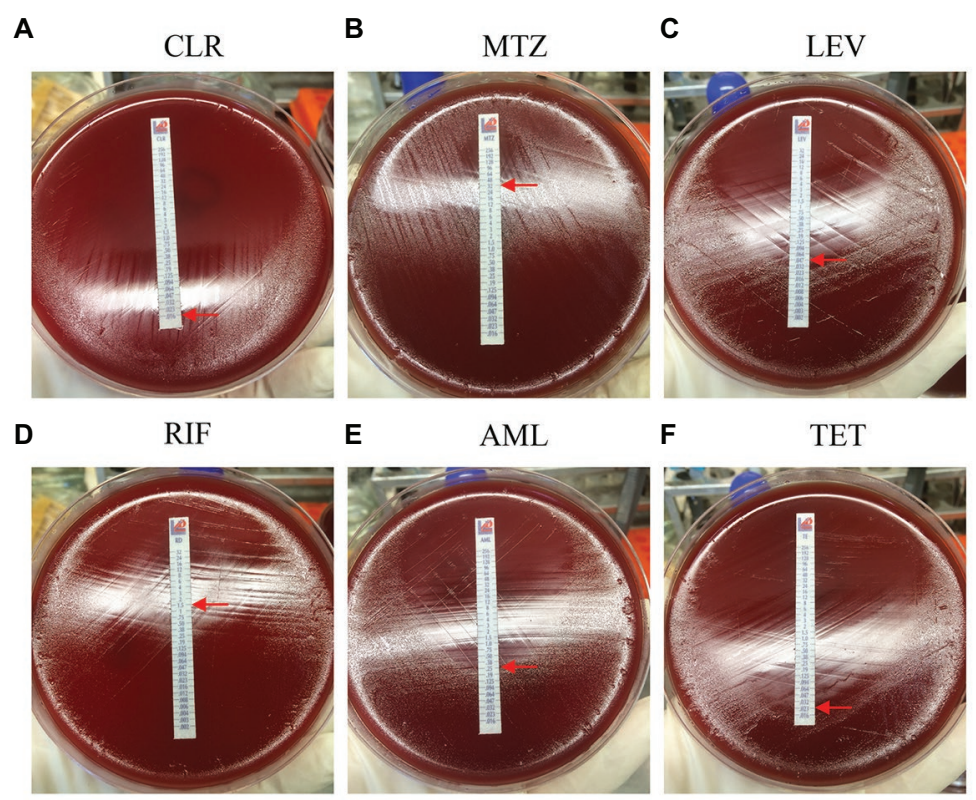

E $\quad$ AML

$\mathbf{F}$

TET
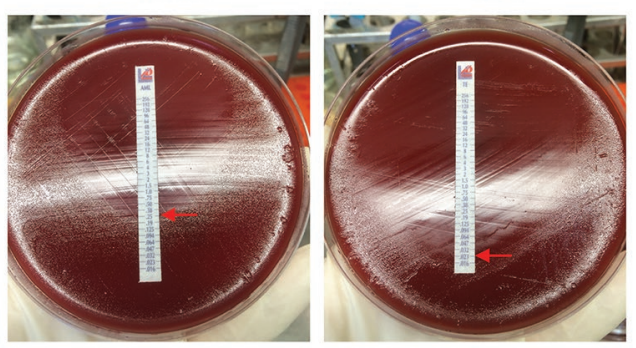

FIGURE 1 | Antibiotic resistance profiles determined by E-test. The minimal inhibitory concentration (MIC) value (the elliptical inhibition zone edge intersecting with the MIC scale on the E-test strip) is indicated by a red arrow. (A) CLR, clarithromycin; (B) MTZ, metronidazole; (C) LEV, levofloxacin; (D) RIF, rifampicin; (E) AML, amoxicillin; and (F) TET, tetracycline. 


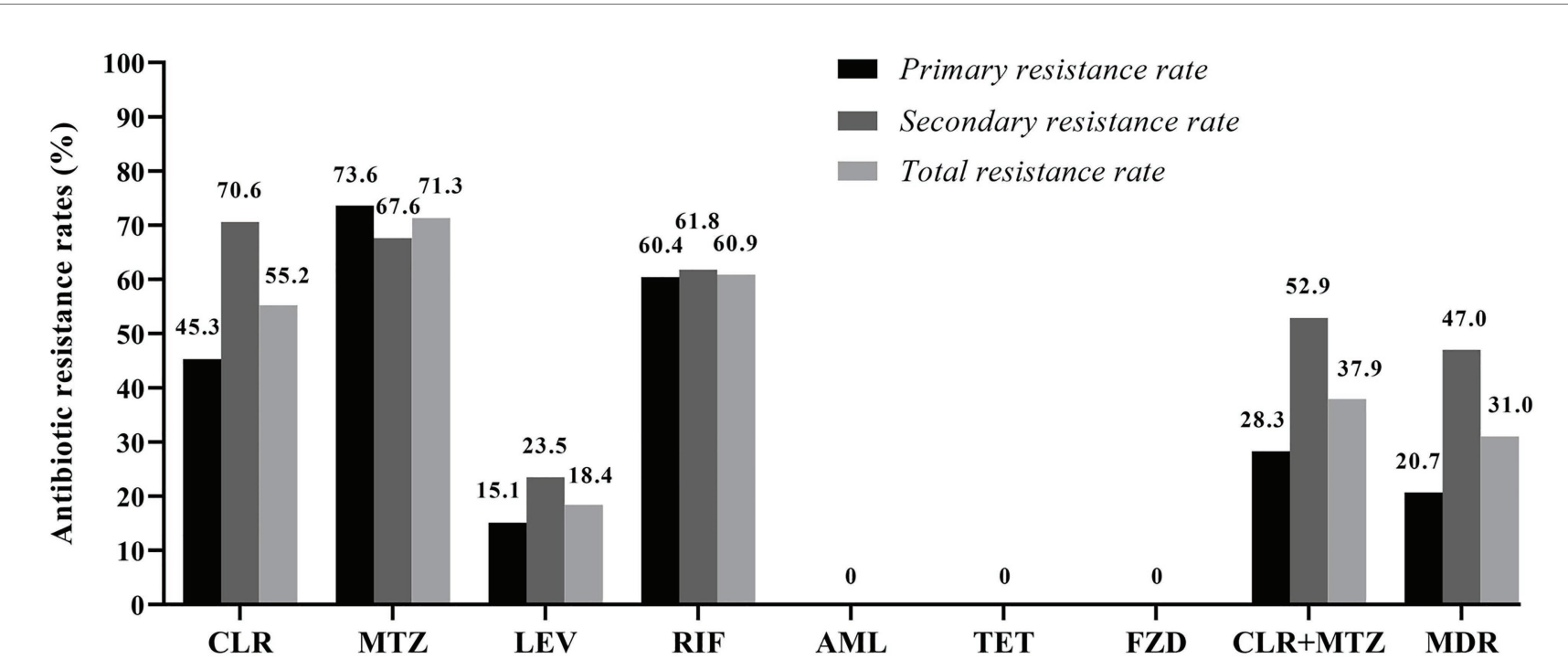

FIGURE 2 | Antibiotic resistance rates among the 87 isolated pediatric strains. CLR, clarithromycin; MTZ, metronidazole; LEV, levofloxacin; RIF, rifampicin; AML, amoxicillin; TET, tetracycline; FZD, furazolidone; and MDR, multidrug resistance. MDR refers strains exhibiting resistance to three or more of the tested antibiotics.

TABLE 2 | Antibiotic resistance patterns among the 87 isolated pediatric strains.

\begin{tabular}{|c|c|c|c|c|c|c|}
\hline \multirow{2}{*}{$\begin{array}{l}\text { Susceptibility testing } \\
\text { results }\end{array}$} & \multicolumn{2}{|c|}{ Treatment-naïve ( $n=53$ ) } & \multicolumn{2}{|c|}{ Treatment-experienced $(n=34)$} & \multicolumn{2}{|c|}{ Overall $(n=87)$} \\
\hline & $n$ & Resistance (\%) & $n$ & Resistance (\%) & $n$ & Resistance (\%) \\
\hline Mono resistance & 12 & 22.6 & 7 & 20.6 & 19 & 21.8 \\
\hline CLR & 5 & 9.4 & 1 & 2.9 & 6 & 6.9 \\
\hline MTZ & 5 & 9.4 & 2 & 5.9 & 7 & 8.0 \\
\hline RIF & 2 & 3.8 & 3 & 8.8 & 5 & 5.7 \\
\hline LEV & 0 & 0 & 1 & 2.9 & 1 & 1.1 \\
\hline Dual resistance & 27 & 50.9 & 10 & 29.4 & 37 & 42.5 \\
\hline$C L R+M T Z$ & 5 & 9.4 & 4 & 11.8 & 9 & 10.3 \\
\hline CLR + RIF & 4 & 7.5 & 3 & 8.8 & 7 & 8.0 \\
\hline LEV + MTZ & 2 & 3.8 & 1 & 2.9 & 3 & 3.4 \\
\hline $\mathrm{RIF}+\mathrm{MTZ}$ & 16 & 30.2 & 2 & 5.9 & 18 & 20.7 \\
\hline Triple resistance & 7 & 13.2 & 15 & 44.1 & 22 & 25.3 \\
\hline $\mathrm{CLR}+\mathrm{MTZ}+\mathrm{RIF}$ & 5 & 9.4 & 10 & 29.4 & 15 & 17.2 \\
\hline $\mathrm{LEV}+\mathrm{MTZ}+\mathrm{RIF}$ & 1 & 1.9 & 0 & 0 & 1 & 1.1 \\
\hline CLR + LEV + RIF & 0 & 0 & 2 & 5.9 & 2 & 2.3 \\
\hline CLR + MTZ + LEV & 1 & 1.9 & 3 & 8.8 & 4 & 4.6 \\
\hline Quadruple resistance & 4 & 7.5 & 1 & 2.9 & 5 & 5.7 \\
\hline $\mathrm{CLR}+\mathrm{MTZ}+\mathrm{LEV}+\mathrm{RIF}$ & 4 & 7.5 & 1 & 2.9 & 5 & 5.7 \\
\hline
\end{tabular}

CLR, clarithromycin; MTZ, metronidazole; LEV, levofloxacin; and RIF, rifampicin.

MTZ and RIF. However, no significant difference in antibiotic resistance rate was observed among the three different ethnic groups. There was no difference between male and female patients in resistance rates to all the seven antibiotics tested in this study. The 34 strains isolated from patients with prior H. pylori treatment were more likely to be resistant to CLR than the 53 strains isolated from $H$. pylori treatment naïve patients (70.6 vs. $45.3 \%, p=0.02$ ). However, no significant difference was observed between treatment-experienced and treatment-naïve patients in resistant rates to MTZ, LEV, and RIF (Table 3).

The distribution of the MIC values against seven antibiotics in 87 pediatric H. pylori strains was shown in Figure 3.
The MIC values against CLR ranged widely: $\leq 0.25 \mathrm{mg} / \mathrm{L}(n=39)$, $1-8(n=19), 12-48(n=20)$, and 64-256 $(n=9)$. Among the 62 MTZ-resistant strains, 79.0\% (49/62) had high level resistance with $\mathrm{MIC} \geq 256 \mathrm{mg} / \mathrm{L}$. All the 71 strains susceptible to $\mathrm{LEV}$ had a low MIC value in the range of $0.012-0.35 \mathrm{mg} / \mathrm{L}$, while among the 16 LEV-resistant strains, $81.3 \%(13 / 16)$ had high level resistance with $\mathrm{MIC} \geq 32 \mathrm{mg} / \mathrm{L}$. The 53 RIF-resistant strains had a wide MIC distribution: $1.5-4 \mathrm{mg} / \mathrm{L}(n=32)$, $6-16 \mathrm{mg} / \mathrm{L}(n=9), 24 \mathrm{mg} / \mathrm{L}(n=1)$, and $\geq 32 \mathrm{mg} / \mathrm{L}(n=11)$. As for the MIC values against AML, more than half of the 87 strains had a MIC $\leq 0.016 \mathrm{mg} / \mathrm{L}(n=49)$, while 29 strains had a MIC in the range of $0.023-0.125 \mathrm{mg} / \mathrm{L}$, eight had a 
TABLE 3 | Factors associated with Helicobacter pylori resistance to CLR, MTZ, LEV, and RIF in pediatric patients.

\begin{tabular}{|c|c|c|c|c|c|c|c|c|c|}
\hline & $n$ & $\begin{array}{c}\text { CLR } \\
\text { resistance }\end{array}$ & $p$ & $\begin{array}{c}\text { MTZ } \\
\text { resistance }\end{array}$ & $p$ & $\begin{array}{c}\text { LEV } \\
\text { resistance }\end{array}$ & $p$ & $\begin{array}{c}\text { RIF } \\
\text { resistance }\end{array}$ & $p$ \\
\hline \multicolumn{10}{|l|}{ Age } \\
\hline $5-11$ & 47 & 27 (57.4\%) & 0.98 & 35 (74.5\%) & 0.47 & 12 (25.5\%) & 0.06 & 27 (57.4\%) & 0.47 \\
\hline $12-17$ & 40 & $21(52.5 \%)$ & & 27 (67.5\%) & & $4(10.0 \%)$ & & 26 (65.0\%) & \\
\hline \multicolumn{10}{|c|}{ Race/ethnics } \\
\hline Han & 59 & $36(61.0 \%)$ & 0.10 & 45 (76.3\%) & 0.15 & 12 (20.3\%) & 0.25 & 35 (59.3\%) & 0.58 \\
\hline Tibetan & 22 & 11 (50.0\%) & & 12 (54.5\%) & & 2 (9.1\%) & & 13 (59.1\%) & \\
\hline Yi & 6 & $1(16.7 \%)$ & & 5 (83.3\%) & & $2(33.3 \%)$ & & 5 (83.3\%) & \\
\hline \multicolumn{10}{|c|}{ Gender } \\
\hline male & 53 & 25 (47.2\%) & 0.06 & 38 (71.7\%) & 0.91 & $9(17.0 \%)$ & 0.67 & 33 (62.3\%) & 0.75 \\
\hline female & 34 & $23(67.6 \%)$ & & 24 (70.6\%) & & 7 (20.6\%) & & 20 (58.8\%) & \\
\hline \multicolumn{10}{|c|}{ Prior treatment } \\
\hline No & 53 & 24 (45.3\%) & 0.02 & 39 (73.6\%) & 0.55 & $8(15.1 \%)$ & 0.32 & 32 (60.4\%) & 0.90 \\
\hline Yes & 34 & $24(70.6 \%)$ & & 23 (67.6\%) & & 8 (23.5\%) & & 21 (61.8\%) & \\
\hline
\end{tabular}

CLR, clarithromycin; MTZ, metronidazole; LEV, levofloxacin; and RIF, rifampicin.

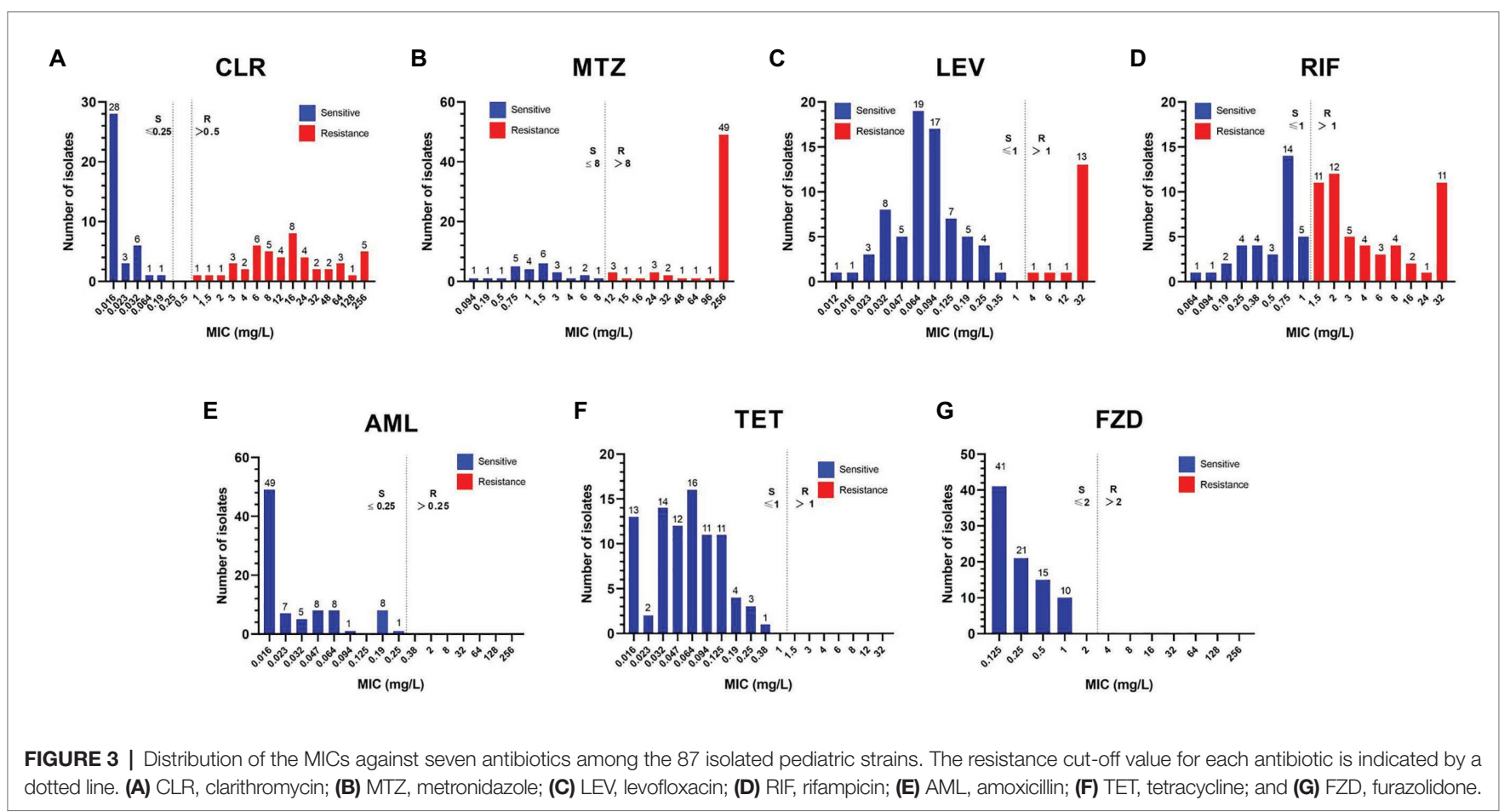

MIC value at $0.19 \mathrm{mg} / \mathrm{L}$, and the remaining one strain had a MIC value at $0.25 \mathrm{mg} / \mathrm{L}$. All the 87 strains were susceptible to TET and FZD. The MIC values against TET were $\leq 0.125 \mathrm{mg} / \mathrm{L}$ in more than $90 \%$ of the strains (79/87), and nearly half of the strains tested against FZD had a MIC $\leq 0.125 \mathrm{mg} / \mathrm{L}(n=41)$.

\section{DISCUSSION}

Helicobacter pylori infection is a bacterial infectious disease and therefore successful $H$. pylori eradication is mainly dependent on the choice of susceptible antibiotics. However, since antimicrobial susceptibility testing for $H$. pylori is almost universally unavailable, it has been recommended that the choice of an effective empirical eradication therapy is based on region and population-specific antibiotic resistance patterns. With $87 \mathrm{H}$. pylori pediatric strains isolated in Southwest China from three different ethnic groups (Han, Tibetan, and Yi), we demonstrated a very high overall resistance rate to CLR (55.2\%), MTZ (71.3\%), and RIF (60.9\%). The overall resistance rate to LEV was $18.4 \%$. None of these pediatric strains were resistant to AML, TET, and FZD.

To our knowledge, this is the first study to report the antibiotic susceptibility profile among pediatric patients in Southwest China. The primary CLR resistance among the 53 treatment-naïve pediatric patients was $45.3 \%$ in this study, 
which was much higher than the primary CLR resistance rates reported among pediatric patients in Southeast China including Zhejiang province (16.4\%; Li et al., 2017) and Jiangxi province (22\%; Liu et al., 2019). Interestingly, the primary CLR resistance rate among pediatric patients in Beijing (northern China) was reported as high as $84.9 \%$ in 2011 (Liu et al., 2011). In view of the extremely high primary and secondary CLR resistance, the CLR-based regimens (even with the addition of bismuth) are no longer suitable as empiric treatment therapies for pediatric patients in Southwest China. However, of note is that if CLR is known to be susceptible by antimicrobial susceptibility testing, this antibiotic can be confidently prescribed to achieve successful $H$. pylori eradication (Jones et al., 2017; Tang et al., 2020a).

The recorded primary MTZ resistance among the pediatric population examined was $73.6 \%$, which was much higher than the primary MTZ resistance reported among pediatric patients in Jiangxi province (46\%; Liu et al., 2019), but it is comparable to the regional MTZ resistance rate recently reported among pediatric patients in Zhejiang province (75.2\%; Li et al., 2017). Simultaneous resistance to CLR and MTZ was present in $28.3 \%$ of the 53 treatment-naïve patients, and in $52.9 \%$ of the 34 pediatric patients with previous treatment in this study, suggesting that the concomitant regimen (PPI, AML, CLR, and MTZ) might not be suitable as empiric therapy for these pediatric patients. For $H$. pylori eradication in adults, the MTZ resistance can be well overcome by increasing the dosage of MTZ or the dosage of AML (Liang et al., 2013; Zhang et al., 2015; Chen et al., 2019). Whether increasing the dosage of MTZ or AML can overcome MTZ resistance has not been studied in pediatric patients. Interestingly, it has been reported that among pediatric patients in Japan with MTZ resistance surpassing 20\% (Okuda et al., 2017), the standard triple therapy with the combination of PPI, AML, and MTZ can still achieve an excellent eradication rate of more than 95\% (Okuda et al., 2017; Mabe et al., 2018). This suggests that low level MTZ resistance may exert very limited influence on $H$. pylori eradication. However, considering the high MTZ resistance rate observed in this study, the bismuth quadruple therapy through the addition of bismuth to the standard triple therapy (PPI, AML, and MTZ) would be suitable as first-line empiric treatment regimen for pediatric patients in China. The major role of bismuth is to increase an additional $30-40 \%$ eradication success for resistant $H$. pylori infections. Of note is that bismuth in China has only been recommended for patients older than 6 years (Xu et al., 2015).

The use of quinolones is contraindicated in patients younger than 18 years in China. However, the primary LEV resistance rate among the pediatric patients in this study was $15.1 \%$, which seemed higher than the primary LEV resistance rate reported in Zhejiang province (6.7\%), but it is comparable to the primary LEV resistance reported in Beijing (13.7\%). As $H$. pylori infection is usually acquired in childhood mainly through intra-family transmission, the high LEV resistance in children might be explained by the transmission of LEV-resistant strains from parents to children.
RIB has been recommended for refractory $H$. pylori treatment (Gisbert and Calvet, 2012; Fallone et al., 2016; Chey et al., 2017; Malfertheiner et al., 2017), while in the laboratory, the MICs of RIF are used for screening RIB resistance (Hays et al., 2018). In this study, both primary and secondary RIF resistance rates surpassed $60 \%$. Of the 53 RIF-resistant strains, 11 strains exhibited high level resistance to RIF with MIC values being recorded $\geq 32 \mathrm{mg} / \mathrm{L}$ (Figure $\mathbf{1}$ ). The extremely high RIF resistance might be partly ascribed to the frequent use of RIF/RIB for the treatment of Mycobacterium tuberculosis, which remains a serious public health problem in Southwest China.

Resistance to AML was not detected among the pediatric patients in this study, which was in line with the negligible AML resistance rate $(0.06 \%)$ reported among pediatric patients in Zhejiang province (Li et al., 2017), and the nil AML resistance among pediatric patients in Beijing (Liu et al., 2011). AML resistance in children has also been reported as nil or negligible in many other countries, including Portugal ( $0.0 \%$; Silva et al., 2018), Spain (0.0\%; Montes et al., 2015), Germany (0.8\%; Regnath et al., 2017), Italy (0.0\%; Manfredi et al., 2015), and Poland (0.0\%; Oleastro et al., 2011). As none of strains isolated from the treatment-experienced pediatric patients were found to be resistant to AML, it is not necessary to consider AML resistance in patients with previous treatment failures. Nil resistance to TET and FZD was also observed among the pediatric patients in this study. However, due to the side effects of teeth discoloration and enamel hypoplasia, TET is contraindicated in children younger than 8 years. The use of FZD is only indicated for the treatment of refractory $H$. pylori infections. Of note, FZD is forbidden for use in pediatric patients younger than 14 years.

Our study has several limitations. First, we only used the classical phenotypic antimicrobial susceptibility testing method to assess the antibiotic resistance profile in this study. Genotypic molecular tests need to be considered in future studies for the detection of CLA and LEV resistance. Second, our study was conducted among pediatric patients in a single tertiary hospital, and so our findings may not be applied in the general pediatric patients in Southwest China. Third, the number of pediatric patients included in this study was relatively small, which might be the reason of not being able to detect the difference in antibiotic resistance rate among different ethnic groups. Future studies are needed to include more pediatric patients to investigate factors (e.g., age, race, gender, and prior treatment history) that are likely to influence antibiotic resistance profiles in pediatric $H$. pylori strains.

In summary, we demonstrated very high antibiotic resistance rates to CLR, MTZ, LEV, and RIF in this study, whereas resistance to AML, TET, and FZD was not detected. Our data suggest that the standard CLR-based triple therapy should be abandoned as empiric therapy for pediatric patients in Southwest China. AML should be an indispensable antibiotic component of the initial or rescue $H$. pylori eradication regimens provided that the pediatric patients are not allergic to AML. Considering the extremely high CLR and MTZ resistance, and the limited choice of antibiotics for H. pylori treatment in children, TET and FZD may be considered for treating refractory $H$. pylori infections in pediatric patients older than 8- and 14-years. 


\section{DATA AVAILABILITY STATEMENT}

The original contributions presented in the study are included in the article/supplementary material, further inquiries can be directed to the corresponding authors.

\section{ETHICS STATEMENT}

The studies involving human participants were reviewed and approved by Ethics Committee of West China Second Hospital of Sichuan University. Written informed consent to participate in this study was provided by the participants' legal guardian/next of kin.

\section{AUTHOR CONTRIBUTIONS}

HL worked on design and concept of this work, drafting, and revising the manuscript. JL, JD, and $\mathrm{ZW}$ implemented this

\section{REFERENCES}

Chen, Q., Long, X., Ji, Y., Liang, X., Li, D., Gao, H., et al. (2019). Randomised controlled trial: susceptibility-guided therapy versus empiric bismuth quadruple therapy for first-line Helicobacter pylori treatment. Aliment. Pharmacol. Ther. 49, 1385-1394. doi: 10.1111/apt.15273

Chey, W. D., Leontiadis, G. I., Howden, C. W., and Moss, S. F. (2017). ACG clinical guideline: treatment of Helicobacter pylori infection. Am. J. Gastroenterol. 112, 212-239. doi: 10.1038/ajg.2016.563

Fallone, C. A., Chiba, N., Van Zanten, S. V., Fischbach, L., Gisbert, J. P., Hunt, R. H., et al. (2016). The Toronto consensus for the treatment of Helicobacter pylori infection in adults. Gastroenterology 151, 51.e14-69.e14. doi: 10.1053/j.gastro.2016.04.006

Gisbert, J. P., and Calvet, X. (2012). Review article: rifabutin in the treatment of refractory Helicobacter pylori infection. Aliment. Pharmacol. Ther. 35, 209-221. doi: 10.1111/j.1365-2036.2011.04937.x

Graham, D. Y., Canaan, Y., Maher, J., Wiener, G., Hulten, K. G., and Kalfus, I. N. (2020). Rifabutin-based triple therapy (RHB-105) for Helicobacter pylori eradication: a double-blind, randomized, controlled trial. Ann. Intern. Med. 172, 795-802. doi: 10.7326/M19-3734

Hays, C., Burucoa, C., Lehours, P., Tran, C. T., Leleu, A., and Raymond, J. (2018). Molecular characterization of Helicobacter pylori resistance to rifamycins. Helicobacter 23. doi: 10.1111/hel.12451

Jones, N. L., Koletzko, S., Goodman, K., Bontems, P., Cadranel, S., Casswall, T., et al. (2017). Joint ESPGHAN/NASPGHAN guidelines for the management of Helicobacter pylori in children and adolescents (update 2016). J. Pediatr. Gastroenterol. Nutr. 64, 991-1003. doi: 10.1097/MPG.0000000000001594

Kahlmeter, G., Brown, D. F., Goldstein, F. W., Macgowan, A. P., Mouton, J. W., Odenholt, I., et al. (2006). European committee on antimicrobial susceptibility testing (EUCAST) technical notes on antimicrobial susceptibility testing. Clin. Microbiol. Infect. 12, 501-503. doi: 10.1111/j.1469-0691.2006.01454.x

Li, L., Ke, Y., Yu, C., Li, G., Yang, N., Zhang, J., et al. (2017). Antibiotic resistance of Helicobacter pylori in Chinese children: a multicenter retrospective study over 7 years. Helicobacter 22. doi: 10.1111/hel.12373

Li, H., Yang, T., Tang, H., Tang, X., Shen, Y., Benghezal, M., et al. (2019). Helicobacter pylori infection is an infectious disease and the empiric therapy paradigm should be changed. Precis. Clin. Med. 2, 77-80. doi: 10.1093/ pcmedi/pbz009

Liang, X., Xu, X., Zheng, Q., Zhang, W., Sun, Q., Liu, W., et al. (2013). Efficacy of bismuth-containing quadruple therapies for clarithromycin-, metronidazole-, and fluoroquinolone-resistant Helicobacter pylori infections in a prospective study. Clin. Gastroenterol. Hepatol. 11, 802.e801-807.e801. doi: 10.1016/j. cgh.2013.01.008 study and analysis of data. CW provided the funding support and project administration. All authors contributed to the article and approved the submitted version.

\section{FUNDING}

This work was supported by the Research and Development Project of Sichuan Provincial Science and Technology Department (2018SZ0130 and 2020JDKP0015), and by the 1.3.5 Project for Disciplines of Excellence, West China Hospital, Sichuan University (no. ZY2016201).

\section{ACKNOWLEDGMENTS}

We thank Yalin Shen for providing technical assistance in isolation, culture and antimicrobial susceptibility testing of pediatric H. pylori isolates in this study.

Liu, D. S., Wang, Y. H., Zhu, Z. H., Zhang, S. H., Zhu, X., Wan, J. H., et al. (2019). Characteristics of Helicobacter pylori antibiotic resistance: data from four different populations. Antimicrob. Resist. Infect. Control 8:192. doi: 10.1186/s13756-019-0632-1

Liu, W. Z., Xie, Y., Lu, H., Cheng, H., Zeng, Z. R., Zhou, L. Y., et al. (2018). Fifth Chinese National Consensus Report on the management of Helicobacter pylori infection. Helicobacter 23:e12475. doi: 10.1111/ hel.12475

Liu, G., Xu, X., He, L., Ding, Z., Gu, Y., Zhang, J., et al. (2011). Primary antibiotic resistance of Helicobacter pylori isolated from Beijing children. Helicobacter 16, 356-362. doi: 10.1111/j.1523-5378.2011.00856.x

Mabe, K., Okuda, M., Kikuchi, S., Amagai, K., Yoshimura, R., Kato, M., et al. (2018). Randomized controlled trial: PPI-based triple therapy containing metronidazole versus clarithromycin as first-line treatment for Helicobacter pylori in adolescents and young adults in Japan. J. Infect. Chemother. 24, 538-543. doi: 10.1016/j.jiac.2018.02.013

Malfertheiner, P., Megraud, F., O’morain, C. A., Gisbert, J. P., Kuipers, E. J., Axon, A. T., et al. (2017). Management of Helicobacter pylori infection-the Maastricht V/Florence consensus report. Gut 66, 6-30. doi: 10.1136/ gutjnl-2016-312288

Manfredi, M., Gismondi, P., Maffini, V., Bizzarri, B., Fornaroli, F., Madia, C., et al. (2015). Primary antimicrobial susceptibility changes in children with Helicobacter pylori infection over 13 years in northern Italy. Gastroenterol. Res. Pract. 2015:717349. doi: 10.1155/2015/717349

Montes, M., and Pérez-Trallero, E. (2017). How long until routine Helicobacter pylori antimicrobial susceptibility testing? Lancet Infect. Dis. 17, 130-131. doi: 10.1016/s1473-3099(17)30005-1

Montes, M., Villalon, F. N., Eizaguirre, F. J., Delgado, M., Munoz-Seca, I. M., Fernandez-Reyes, M., et al. (2015). Helicobacter pylori infection in children. Antimicrobial resistance and treatment response. Helicobacter 20, 169-175. doi: $10.1111 /$ hel.12187

Okuda, M., Kikuchi, S., Mabe, K., Osaki, T., Kamiya, S., Fukuda, Y., et al. (2017). Nationwide survey of Helicobacter pylori treatment for children and adolescents in Japan. Pediatr. Int. 59, 57-61. doi: 10.1111/ped.13038

Oleastro, M., Cabral, J., Ramalho, P. M., Lemos, P. S., Paixao, E., Benoliel, J., et al. (2011). Primary antibiotic resistance of Helicobacter pylori strains isolated from Portuguese children: a prospective multicentre study over a 10 year period. J. Antimicrob. Chemother. 66, 2308-2311. doi: 10.1093/jac/ dkr293

Regnath, T., Raecke, O., Enninger, A., and Ignatius, R. (2017). Increasing metronidazole and rifampicin resistance of Helicobacter pylori isolates obtained from children and adolescents between 2002 and 2015 in Southwest Germany. Helicobacter 22. doi: 10.1111/hel.12327 
Silva, G. M., Silva, H. M., Nascimento, J., Goncalves, J. P., Pereira, F., and Lima, R. (2018). Helicobacter pylori antimicrobial resistance in a pediatric population. Helicobacter 23:e12528. doi: 10.1111/hel.12528

Su, P., Li, Y., Li, H., Zhang, J., Lin, L., Wang, Q., et al. (2013). Antibiotic resistance of Helicobacter pylori isolated in the southeast coastal region of China. Helicobacter 18, 274-279. doi: 10.1111/hel.12046

Sugano, K., Tack, J., Kuipers, E. J., Graham, D. Y., El-Omar, E. M., Miura, S., et al. (2015). Kyoto global consensus report on Helicobacter pylori gastritis. Gut 64, 1353-1367. doi: 10.1136/gutjnl-2015-309252

Tang, H., Li, H., Marshall, B. J., Benghezal, M., Stubbs, K. A., Debowski, A. W., et al. (2020a). Susceptibility-guided bismuth quadruple therapies for resistant Helicobacter pylori infections. Precis. Clin. Med. 3, 127-135. doi: 10.1093/ pcmedi/pbaa010

Tang, X., Shen, Y., Hu, R., Yang, T., Benghezal, M., Li, H., et al. (2020b). Re-assessment of the disk diffusion technique for routine antimicrobial susceptibility testing for Helicobacter pylori. Helicobacter 25:e12703. doi: 10.1111/hel.12703
Xu, C., Li, H., Gong, S., Geng, L., Zhao, J., Sun, M., et al. (2015). Expert consensus on diagnosis and treatment of Helicobacter pylori infection in children. Chin. J. Prediatr. 7, 496-498. doi: 10.3760/cma.j.issn.0578-13/0.2015.07.006

Zhang, W., Chen, Q., Liang, X., Liu, W., Xiao, S., Graham, D. Y., et al. (2015) Bismuth, lansoprazole, amoxicillin and metronidazole or clarithromycin as firstline Helicobacter pylori therapy. Gut 64, 1715-1720. doi: 10.1136/gutjnl-2015-309900

Conflict of Interest: The authors declare that the research was conducted in the absence of any commercial or financial relationships that could be construed as a potential conflict of interest.

Copyright (c) 2021 Li, Deng, Wang, Li and Wan. This is an open-access article distributed under the terms of the Creative Commons Attribution License (CC BY).

The use, distribution or reproduction in other forums is permitted, provided the original author(s) and the copyright owner(s) are credited and that the original publication in this journal is cited, in accordance with accepted academic practice. No use, distribution or reproduction is permitted which does not comply with these terms. 